\title{
EL ESTATUS CATEGORIAL DE PUROS PRENOMINAL: UN DETERMINANTE IMPROBABLE
}

\section{INTRODUCCIÓN ${ }^{1}$}

En español mexicano ${ }^{2}$, la expresión puros se emplea antepuesta al sustantivo para proferir un significado que, intuitivamente, es muy parecido al de sólo o solamente. Así, en el ejemplo (1) se asevera que los encargados de arar son exclusivamente tractores y en (2) se afirma que lo único que se oye son malas palabras. En (3) se describe la situación de que quienes comandan al ejército son generalitos de banqueta, y se excluye que tengan otra propiedad relevante. De manera informal, podemos decir que puros antepuesto introduce una operación de exhaustividad que implica la exclusión de alternativas:

1 Agradezco a Josefina García Fajardo, Sergio E. Bogard Sierra, Julia Pozas Loyo, Ana Aguilar Guevara y a dos dictaminadores anónimos los comentarios y sugerencias que han vertido sobre este trabajo. Los errores y omisiones que en él se encuentren son responsabilidad mía.

${ }^{2}$ Este uso no es exclusivo del español mexicano. Al parecer, está extendido por Hispanoamérica, aunque no me es posible saber si en otros dialectos tiene, además, otras implicaciones como, por ejemplo, un significado parecido al de muchos. Por ello, en este trabajo me limito a describir datos del español mexicano, ya sea ejemplificando con oraciones encontradas en corpus, en Google, o con ejemplos propios. Los corpus empleados para obtener algunos ejemplos son los siguientes: DiCGIONARIO DEL EsPAÑOL DE MÉxico. Corpus del español mexicano contemporáneo (CEMC). <http:/ /www. corpus.unam.mx/cemc $>$, software AMATE ver. 1.0, [última consulta 02-102014]; Real ACAdemia Española: Banco de datos (CREA) [en línea]. Corpus de referencia del español actual. <http:/ / www.rae.es $>$ [última consulta 02-102014]; Pedro Martín Butragueño y Yolanda Lastra (coords.), Corpus sociolingüistico de la Ciudad de México. T. 1: Nivel alto, El Colegio de México, México, 2011 (CSCM). 
(1) Los bueyes ya casi no se usa. Puros tractores son los que aran todo eso (CEMC, 1970, Anónimo, Cinta 3-II-A).

(2) Nomás que en lugar de rezos se oyen puras malas palabras (CEMC, 1970, Armida de la Vara, Fragmentos I).

(3) los que ahora están al frente del ejército son puros generalitos de banqueta (CREA, 1983, Arturo Azuela, La casa de las mil vírgenes).

En este artículo, describiré la distribución sintáctica y la interpretación de las frases nominales introducidas por puros y sin otros determinantes. Argumentaré que, semánticamente, estas frases se comportan como plurales escuetos en el sentido de que no toman alcance sobre otros operadores (lo que se conoce como "alcance inerte"), ni tampoco tienen interpretación referencial, y eso lleva a pensar que puros no es otra cosa que un modificador adjetival. Pero, por otro lado, también mostraré que su distribución sintáctica es parecida a la que se espera de los determinantes, pues puros legitima sujetos preverbales y, en ciertas construcciones, categorías vacías. Este comportamiento híbrido nos lleva a indagar sobre el estatus categorial de esta pieza léxica, y propondré que, como determinante, infringe el universal de conservatividad (que explicaré más adelante). Por lo tanto, propongo que se trata de un adjetivo de sentido cuantificacional.

Los ejemplos de (1) a (3) nos muestran algunas de las características más evidentes de puros. Primero, que a pesar de su obvia relación con el adjetivo puro, cuyo significado describe el Diccionario del español de México como "Que conserva su naturaleza original, que no está mezclado con nada, que no tiene elementos extraños", puros antepuesto no se predica distributivamente del sustantivo que le sigue. Así, en (1), no se afirma de cada uno de los tractores que sean puros, ni tampoco se obtiene de (2) la idea de que las malas palabras que se oyen sean, a la vez, puras. En segundo lugar, como muestran los ejemplos (1) y (2), las frases nominales con puros no solamente tienen usos predicativos, como en (3), sino también argumentales. En este aspecto, (2) es especialmente revelador, pues el sujeto con puros está antepuesto al verbo, posición típicamente reservada a las frases nominales con determinante.

En tercer lugar, vemos que puros antepuesto, al igual que su contraparte postnominal, concuerda en género y número con 
el sustantivo con el que se combina. Esta concordancia indica que puros está sintácticamente dentro de la frase nominal, y que no tiene el carácter adverbial de las expresiones nomás, sólo o solamente, que también expresan exhaustividad ${ }^{3}$. Algunos trabajos, como los de Gutiérrez-González ${ }^{4}$ y Pato ${ }^{5}$ consideran que puro es un adverbio concordado, como lo sería solo en casos como (5), con base en alternancias como las de $(4)^{6}$ :

(4) a. Dijo la pura verdad.

b. Dijo puro la verdad.

(5) a. La sola mención de su nombre me atemoriza.

$b$. Solo la mención de su nombre me atemoriza.

El hecho de que en (4a) pura tenga alcance sobre la frase nominal, incluyendo al determinante que la precede ${ }^{7}$, sugiere que esta expresión es, en realidad, un adverbio concordado, y que podría serlo también en casos como los de (1)-(3) y otros que se analizan en este trabajo. Sin embargo, hay dos razones por las que encuentro problemática esta hipótesis. La primera es que, al menos a primera vista, es más plausible pensar que el uso adverbial de puro en ( $4 b)$ es efecto de una recategorización del adjetivo y no al revés. Si éste es el caso, para explicar el comportamiento del adverbio debemos primero comprender la semántica del adjetivo adnominal, lo cual nos lleva de vuelta al inicio del planteamiento de este trabajo: cuál es el estatus categorial y la función semántica de puros dentro de la frase nominal. Segundo, el fenómeno de la concordancia adverbial, si bien, como ha mostrado Pato $(i d$.) está más extendido de lo que se piensa, en el caso de puros se restringe a las frases encabezadas por determinante, y no se da en las frases nominales

3 Una prueba más de su carácter adjetival en posición antepuesta es la posibilidad de tomar flexión de diminutivo, como lo hacen los adjetivos, pero muy raramente los adverbios: Lo dejó en los puritos huesos. Incluso se encuentran algunos ejemplos con diminutivos reduplicados: Eso que se cuenta son purititas mentiras de final de sexenio.

4 Yurena Gutiérrez-González, “¿Operadores focales exhaustivos nomás?”, CALFAL, 3 (2011), 42-59.

5 ENRiQue Pato, "La recategorización del adverbio medio en español", BdFs, 45 (2010), 91-110.

${ }^{6}$ Agradezco a un dictaminador anónimo el haberme sugerido esta bibliografía.

7 Así lo sugería un dictaminador anónimo. 
sin determinante, como las que aquí nos ocupan. Los adverbios concordados conservan las condiciones veritativas de sus contrapartes no concordadas, como en el par (5). Sin embargo, cuando la frase nominal carece de determinante, tal equivalencia es imposible de sostener con puros:

(6) a. Vimos puras películas argentinas.

b. ?Puro vimos películas argentinas.

(7) a. Puros burócratas manejan el partido.

b. ?/*Puro manejan burócratas el partido.

En la medida en que las oraciones $(6 b)$ y $(7 b)$ son gramaticales o aceptables -y esto ya es cuestionable-, no son semánticamente equivalentes a sus respectivas contrapartes en $(6 a)$ y $(7 a)$, lo que me lleva a cuestionar que puros en esas oraciones formalice un adverbio concordado. No descarto esa posibilidad para las ocurrencias de puro/pura, puros/puras dentro de las frases encabezadas con determinante, pero, en todo caso, considero que defender que se trate de adverbios concordados en esos casos es una postura que amerita argumentación. Ese objetivo queda fuera del alcance del presente trabajo, en el que me limito a considerar frases nominales sin determinante en las que puros es un elemento interno de la frase nominal.

Por la concordancia de número, vemos que puro(s) aparece como contable plural o como continuo, dependiendo del carácter del sustantivo con el que se combina. Así, se puede encontrar la expresión puro con sustantivos continuos, como en (8), pero si antecede a un sustantivo contable singular, éste se reinterpreta como continuo y abstracto, como en (9), donde el sustantivo tractor refiere a una función o propiedad y no a un objeto contable concreto. Puro sin marca de plural también puede introducir sustantivos colectivos, como gente en el ejemplo (10):

(8) Se quitan todos los dolores. Pura medicina tomo (CEMC, 1975, Anónimo, Cinta 4-I-B).

(9) Aquí es puro tractor ya. Toda esta parte de aquí, puros tractores son los que trabajan ya (CECM, 1970, Anónimo, Cinta 3-II-A).

(10) Ya gente de por aquí, casi no hay. Casi pura gente de fuera es la que está aquí (CECM, 1968, Anónimo, Cinta 1-I-A). 
A menos que vaya precedido de un determinante singular, puro antepuesto no se combina con sustantivos contables en singular, pues si lo hace, los recategoriza en sustantivos continuos, como en (9). En esto también contrasta el adjetivo prenominal con su versión posnominal, pues éste último no tiene restricciones para combinarse con sustantivos contables en singular (alma pura), en plural (almas puras), con sustantivos continuos (aire puro) y con colectivos (gente pura).

Se puede encontrar la expresión puro(s) empleada con un sentido minusvalorativo, ejemplificado en (11). Pero, como ya ha argumentado Fuentes Rodríguez ${ }^{8}$, y como podemos ver en los ejemplos (1), (2) y de (8) a (10), esa inferencia no está necesariamente asociada a todos sus usos, y al parecer, la minusvaloración depende del sustantivo y no del propio adjetivo antepuesto:

(11) Son puros garabatos de esos que dizque llaman ecuaciones (CECM, 1974, Anónimo, El hombre invisible).

En este trabajo me concentraré únicamente en la forma marcada con plural y, de ella, en su función como argumento verbal. Me restrinjo a las frases nominales en función de argumento verbal porque me interesa indagar si el estatus categorial de puros ha pasado de adjetivo a determinante, y para ello es necesario analizar su distribución en posiciones argumentales. Además, escojo solamente la forma plural porque, aunque la forma en singular puro parezca tener un uso más frecuente que la forma plural puros ${ }^{9}$, tiene exclusivamente lecturas colectivas o continuas ${ }^{10}$. Una de las características más típicas de los determinantes es su capacidad de individuación, y esta característica es más clara en los determinantes contables que en los que determinan sustantivos continuos o colectivos.

8 Catalina Fuentes Rodríguez, "Los operadores argumentativos mero, puro, simple y solo", AEF, 25 (2002), 83-101.

${ }^{9}$ Por lo menos así lo sugiere una revisión a primera vista en dos corpus: las concordancias para puros como adjetivo antepuesto en el CECM son 75, frente a 159 para puro en la misma función. En CREA, las concordancias fueron 118 y 271, respectivamente. De estas búsquedas se excluyen, desde luego, los usos de puro y puros como sustantivos y como adjetivos pospuestos, pero también los usos antepuestos precedidos de la expresión de grado más y en coordinación con otro adjetivo, como simple y puro, o tierno y puro.

${ }^{10}$ Como bien lo hace notar un dictaminador anónimo. 
Por último, cabe notar que puros, a pesar de actuar en muchos sentidos como determinante, puede estar precedido por un artículo o un numeral. Sólo en estos casos, si el determinante es singular, el sustantivo puede ser contable sin que la presencia de puro lo recategorice en un sustantivo continuo:

(12) [cuando llegamos aquí no había nada] era un puro cerro aquí (CSCM, Nivel Alto, ME-009-33H-97, 30).

(13) si el puro coche/ el puro carro pesa/ cinco toneladas (CSCM, Nivel Alto, ME-009-33H-97, 188).

(14) No hay a dónde ir aquí, más que a las puras cantinas (CEMC, 1972, Anónimo, Cinta 2-I-B).

Estos casos son llamativos porque la exhaustividad asociada a puro(s) tiene alcance sobre toda la frase encabezada por el determinante (un cerro, el carro, las cantinas). De ese modo, la oración (12) se puede parafrasear como "aquí era sólo un cerro", y (13) sería equivalente a "sólo el coche pesa cinco toneladas”. Esta configuración semántica, en la que el operador de exhaustividad asociado a puros tiene efecto sobre la frase que incluye al determinante, desafía la estructura sintáctica superficial de la frase nominal, pues en ella el determinante precede al operador. Como mencioné antes, no descarto la posibilidad de que en estos casos, en los que puro(s) aparece dentro de frases encabezadas con determinante, estemos tratando con un adverbio concordado, cuya mecánica está todavía por estudiarse exhaustivamente, como ya lo afirman Gutiérrez-González y Pato $^{11}$. Dejaré este tema para futuras investigaciones, y empezaré por analizar solamente las frases con puros sin determinante.

\section{Antecedentes}

Hasta donde sé, no contamos en la bibliografía con una descripción semántica de puros prenominal. Fuentes Rodríguez describe la expresión puro y otros adjetivos originalmente calificativos que en posición prenominal tienen valor de operadores. Propone que las expresiones como simple, mero, solo y puro son operadores argumentativos, a los que asocia con una función "macro-

11 Véanse notas 4 y 5 . 
estructural" o discursiva: su contenido no es el que aporta algo directamente a las condiciones de verdad de la oración, sino que tiene un carácter procedimental orientado a "indicar una enfatización informativa" ${ }^{12}$. Concretamente, puro cumple la función argumentativa de "resaltar el sustantivo e indicar su esencia", es decir, llamar la atención del receptor sobre el contenido del sustantivo para verlo "sin otras cosas" (ibid., p. 96). En resumen, puro antepuesto es un operador argumentativo que focaliza el significado del sustantivo con el que se combina, instruyendo al oyente a que lo considere exento de toda mezcla.

Es razonable pensar que puro y puros tengan un significado parecido en su función no calificativa, de modo que podríamos tratar de adoptar la propuesta de Fuentes Rodríguez $(i d$. al caso de puros y considerar esta expresión como un operador argumentativo. Sin embargo, surgen algunos problemas en este intento. El primero y más evidente es que puros no funciona exclusivamente al nivel "macro-estructural" o del discurso, sino que realmente contribuye a determinar las condiciones de verdad de la oración en la que aparece. Para mostrar esto, compárense las oraciones $(15 a)$ y $(15 b)$ :

(15) a. Hay puros gatos en el asilo.

b. Hay gatos en el asilo.

La oración $(15 b)$ puede ser verdadera en un escenario en el que en el asilo hubiera, además de gatos, algunos perros. En ese mismo escenario, (15a) sería falsa, y eso se debe a la presencia de puros en la frase nominal sujeto. Estoy de acuerdo con Fuentes Rodríguez en que puros (al igual que puro en su estudio) no tiene en $(15 b)$ el contenido léxico que tiene en posición pospuesta (cf. En el asilo hay gatos puros), y que en posición antepuesta adquiere un carácter funcional. El cambio más evidente entre la posición antepuesta y la posición postnominal es que, desde esta última, el significado literal de "Que conserva su naturaleza original, que no está mezclado con nada, que no tiene elementos extraños" (DEM) se aplica distributivamente a cada elemento de la denotación del sustantivo (en el ejemplo, a cada gato), mientras que en la posición antepuesta no tiene esa interpretación distributiva. Esto quiere decir que en la posición prenominal, puros no es un predicado sobre individuos (al

12 Art. cit., p. 84. 
igual que puro en esa posición no es un predicado sobre masas o sustancias), sino que adquiere un tipo funcional más alto, aunque no necesariamente el de operador discursivo. Cuál es exactamente su tipo funcional es parte de lo que trato de desentrañar en este trabajo.

La Nueva gramática de la lengua española $(N G L E)^{13}$ incluye dos breves apartados sobre puro, donde lo trata como adverbio concordado (Lo dijo de pura entrometida) (\$13.8d), como un adverbio parecido a los de foco particularizadores $(\$ 40.9 n)$. En estos casos se puede parafrasear con puramente, solamente o simplemente (cf. Nomás se le iba en puro llorar). A este sentido se le puede añadir el de cuantificador intensivo (escenas imaginadas, edulcoradas, perfeccionadas, reales casi de puro vivirlas y revivirlas a todas horas). No hay, en cambio, en la NGLE una descripción de puro como adjetivo prenominal con el sentido que reconocemos y describimos en este trabajo.

En este artículo mostraré que las frases nominales introducidas por puros se comportan como frases nominales escuetas ${ }^{14}$ en lo que concierne a su interacción con operadores como la negación, los cuantificadores y los operadores modales. Haré explícitas estas comparaciones entre plurales escuetos y frases nominales con puros en la sección siguiente. Al mismo tiempo, las frases nominales con puros, como mostraré en el tercer apartado, pueden cumplir funciones sintácticas que se suponen exclusivas de las frases nominales con determinante, como la de sujeto preverbal y la de legitimador de categorías vacías. Este comportamiento mixto entre adjetivos y determinantes cuantificadores es común y ha sido descrito para varios adjetivos del español ${ }^{15}$; pero el caso de puros reviste un interés especial, que

13 RAE-ASALE, Nueva gramática de la lengua española, Espasa, Madrid, 2009.

14 Emplearé el término "frase nominal escueta" para referir a toda frase nominal que carezca de determinante, ya sea que aparezca o no con modificadores adjetivales. Como me concentro sólo en las que están marcadas con plural, alternativamente uso los términos "plural escueto" o "sustantivo plural escueto".

$15 \mathrm{Al}$ respecto el lector puede consultar, entre otros: MANUEL LeOnetTI, Los cuantificadores, Arco/Libros, Madrid, 2007; Bruno Camus Bergareche, "De adjetivos a cuantificadores. Un fragmento de la historia de los indefinidos románicos", en Actas del XXXVII Simposio Internacional de la Sociedad Española de Lingüistica, eds. Inés Olza Moreno, Manuel Casado Velarde y Ramón González Ruiz, Universidad de Navarra, Pamplona, 2008, pp. 87-95; Julia Pozas Loyo, "Diacronía de cierto", ponencia presentada en el XII Congreso 
consiste en lo siguiente: un cuantificador nominal, cualquiera que sea su categoría sintáctica, invariablemente cuantifica sobre un dominio que corresponde a la denotación del sustantivo que toma como complemento. Ese dominio, pues, constituye la restricción del cuantificador, del que éste toma una parte o la totalidad para después relacionarla con el predicado o el resto de la oración (el alcance nuclear del cuantificador). Pues bien, cuando puros actúa como un cuantificador, su restricción no es el sustantivo con el que se combina, sino el resto de la oración en la que aparece. De este modo, en una oración como los niños comieron puras golosinas, puros no cuantifica sobre el conjunto de las golosinas, sino sobre aquello que comieron los niños, de lo cual predica que se trata, en su totalidad, de golosinas.

A la relación directa entre restricción semántica y primer complemento sintáctico se le conoce como la propiedad de conservatividad de los cuantificadores nominales ${ }^{16} \mathrm{y}$ se ha postulado un universal de acuerdo con el cual todos los determinantes cuantificadores de las lenguas naturales son conservativos ${ }^{17}$. Una de las razones por las que no nos apresuraremos a clasificar puros en la categoría de los cuantificadores determinantes es que, precisamente, estaríamos tratando con un determinante que violaría este universal semántico. Sin embargo, no sería la primera vez que una expresión de significado cuantificacional se enfrentara a ese problema. Se han descrito expresiones en otras lenguas cuyo estatus como determinantes cuantificadores se ve comprometido justamente por tener un carácter no conservativo $^{18}$. En la cuarta sección, propondré un análisis semántico para puros y argumentaré que este análisis

Nacional de la Asociación Mexicana de Lingüística Aplicada, Octubre de 2013; Luis EgUREN y CRISTINA SÁnchez, "Imprecisión extensional e imprecisión intensional: la gramática de cierto", Verba, 34 (2007), 99-124.

${ }_{16}$ Más propiamente, corresponde a la propiedad de "conservatividad en el primer argumento", que es la que se considera universal.

17 No expondré aquí los argumentos formales que sustentan ese universal, que el lector interesado puede consultar en EDWARD KEENAN y JONATHAN STAVI, "A Semantic characterization of natural language determiners", LiP, 9 (1986), 253-326, o en DAG WesterstÅHL, "Logical constants in quantifier languages", LiP, 8 (1985), 387-413.

18 En particular: REgINE ECKARDT, Meaning change in grammaticalization, Oxford University Press, Oxford, 2006; Jan Anderssen, Quantification, misc., tesis doctoral, Universidad de Massachusetts, Amherst, 2011; Helena HerBURGER, What counts: Focus and quantification, M.I.T. Press, Cambridge, MA, 2000; D. WESTERSTÅHL, art. cit. 
se puede extender a esos otros casos sin necesidad de infringir el universal de conservatividad de los determinantes. Concluyo el artículo afirmando que, a pesar de su distribución como determinante cuantificador, puros debe ser analizado como un adjetivo.

\section{LAS FRASES NOMINALES CON PUROS COMO FRASES NOMINALES ESGUETAS: ALGANGE INERTE Y FALTA DE REFERENGIALIDAD}

En esta sección, muestro que las frases nominales introducidas por puros poseen las propiedades de alcance inerte que tienen los sustantivos plurales escuetos, según han descrito B. Laca y L. McNally ${ }^{19}$ : no toman alcance amplio ante la presencia de otro operador, como la negación, los operadores intensionales y los cuantificadores. Por ejemplo, la frase nominal unos libros puede tener alcance sobre o bajo el operador de negación, dando dos posibles lecturas a la oración Juan no encontró unos libros. Si la frase nominal se interpreta con alcance amplio, la lectura es que hay ciertos libros que Juan no encontró, mientras que si tiene alcance estrecho, se lee como que simplemente no hay libros que Juan haya encontrado. En cambio, el plural escueto Juan no encontró libros sólo permite la lectura en la que la negación tiene el alcance amplio y la frase escueta alcance estrecho ('No hay libros que haya encontrado Juan'). Esta falta de interacción de alcance entre el plural escueto y la negación es una muestra de su alcance inerte. Además del alcance inerte, otro rasgo común a las frases nominales con puros y los plurales escuetos estriba en que ninguno de ellos puede tener usos anafóricos. En la segunda, describo las posibles interpretaciones de las frases nominales con puros respecto a la negación, los cuantificadores y los operadores modales. Más adelante, argumento que, dada su incapacidad de retomar referentes previamente introducidos en el discurso, las frases nominales con puros, al igual que los plurales sin determinante, no son referenciales.

19 BREnda LACA, "Acerca de la semántica de los 'plurales escuetos' del español”, en El sustantivo sin determinación. La ausencia de determinación en la lengua española, ed. I. Bosque, Visor, Madrid, 1996, pp. 241-268; Louise MaNally, "Bare plurals in Spanish are interpreted as properties", CatJL, 3 (2004), 115-133. 


\section{Negación y cuantificadores}

Es bien sabido que cuando un plural escueto va precedido de una negación, el plural escueto queda siempre bajo su alcan$\mathrm{ce}^{20}$, como se muestra en (15). La oración (15a) es equivalente, en términos veritativo-condicionales, a (16b), pero no a (16c), pues en esa oración el plural escueto está fuera del alcance de la negación. Con el signo \# marco la falta de equivalencia veritativo-condicional de $(16 c)$ respecto a $(16 a)$ :

(16) a. Los turistas no visitaron haciendas.

b. No hubo haciendas que visitaran los turistas.

c. \#Hubo haciendas que no visitaron los turistas.

Las frases nominales introducidas por puros tienen un comportamiento similar. La frase nominal puras haciendas en (17a) se interpreta sólo bajo el alcance de la negación, por lo que las condiciones de verdad de $(17 a)$ y $(17 b)$ son equivalentes: ambas oraciones son verdaderas si, además de haciendas, los turistas visitaron otros sitios. En cambio, (17c) no representa una posible interpretación de $(17 a)$, pues en esta oración el operador de exhaustividad asociado a puros (expresado en $17 c$ con sólo) está fuera del alcance de la negación:

(17) a. Los turistas no visitaron puras haciendas.

b. Los turistas no visitaron sólo haciendas.

c. \#Sólo haciendas no visitaron los turistas.

Los plurales escuetos y las frases nominales con puros contrastan, por su alcance inerte, con las frases nominales indefinidas, que tienen alcance variable. En (18a), ejemplificamos esta propiedad con la frase nominal tres haciendas, que al interactuar con la negación arroja dos posibles interpretaciones. En una de ellas, tres haciendas se interpreta bajo el alcance de la negación. En ese caso, (18a) es equivalente a $(18 b)$ o $\left(18 b^{\prime}\right)$. En la otra lectura, la frase numeral escapa al ámbito de la negación y resulta en una lectura equivalente a la de (18c):

${ }^{20}$ B. LACA, art. cit., pp. 252-253. Para mantener un punto adecuado de comparación, siempre ejemplificaré las propiedades de alcance con frases nominales con puros- $N$ postverbal, pues los plurales escuetos no pueden aparecer en posiciones pre-verbales (salvo que no sean focos o tópicos contrastivos). 
(18) a. Los turistas no visitaron tres haciendas.

b. No hubo tres haciendas que visitaran los turistas

$b$. No fueron tres las haciendas visitadas por los turistas.

c. Hubo tres haciendas que no visitaron los turistas.

El patrón se repite al interactuar con cuantificadores, ante los cuales ni las frases nominales con puros ni los plurales escuetos pueden tomar alcance amplio. Una frase nominal indefinida, como se sabe, sí puede hacerlo. La oración (19a) es equivalente a la oración (19b), pues en ambas el plural escueto cae bajo el alcance de la frase cuantificada tres turistas. En contraste, no hay una lectura de $(19 a)$ que corresponda a las condiciones de verdad de (19c). Una vez más, el signo \# en (19c) marca que esta oración no es una paráfrasis posible de $(19 a)$ :

(19) a. Tres turistas visitaron haciendas.

b. Hubo tres turistas que visitaron haciendas.

c. \# Hubo haciendas que visitaron tres turistas.

En (20) se muestra que el operador de exhaustividad asociado a puros se interpreta bajo el alcance de tres, como se ve en la equivalencia veritativa entre $(20 a)$ y su paráfrasis en $(20 b)$. Si este operador se interpretara fuera del alcance del cardinal, podríamos obtener una lectura de $(20 a)$ parecida a la de la oración $(20 c)$, pero éste no es el caso:

(20) a. Tres turistas visitaron puras haciendas.

b. Hubo tres turistas que visitaron sólo haciendas.

c. \#Sólo hubo haciendas visitadas por tres turistas (p.ej. los museos fueron visitados por cien turistas y los monumentos fueron visitados por veinte).

Las series de oraciones en (21) y (22) muestran una interacción similar entre las frases nominales con puros y los plurales escuetos, respectivamente, con un cuantificador universal:

(21) a. Todos los turistas visitaron haciendas.

b. Todos los turistas son tales que visitaron haciendas.

c. \#Hubo haciendas visitadas por todos los turistas.

(22) a. Todos los turistas visitaron puras haciendas.

b. Todos los turistas son tales que visitaron sólo haciendas. 
c. \#Sólo hubo haciendas visitadas por todos los turistas (p. ej. los museos y los monumentos no fueron visitados por todos los turistas).

Como es sabido, las frases nominales indefinidas por lo general sí tienen alcance variable respecto a los cuantificadores universales y cardinales. Las oraciones $(23 a)$ y $(24 a)$ tienen dos lecturas posibles, equivalentes a las de los incisos $(b)$ y $(c)$ de cada serie:

(23) a. Tres turistas visitaron dos haciendas.

$b$. Hubo tres turistas que visitaron dos haciendas (posiblemente diferentes pares de haciendas).

c. Hubo dos haciendas que visitaron tres turistas.

(24) a. Todos los turistas visitaron una hacienda.

b. Todos los turistas visitaron al menos una hacienda (y posiblemente visitaron distintas haciendas cada uno).

c. Hubo una hacienda que visitaron todos los turistas.

Ni las frases nominales escuetas ni las frases nominales con puros pueden tener alcance amplio respecto a los modales aléticos, como se muestra en (25) y (26) con el adverbio modal 'posiblemente'. Una vez más, la marca \# en (25c) y (26c) señala que esa oración no es una paráfrasis posible de la oración $(a)$ de cada serie. (27a), en cambio, tiene dos posibles interpretaciones dependiendo del alcance que tome la frase nominal indefinida:

(25) a. A la asamblea posiblemente vendrán abogados.

b. Es posible que vengan abogados a la asamblea.

c. \#Hay abogados tales que es posible que vengan a la asamblea.

(26) a. A la asamblea posiblemente vendrán puros abogados.

$b$. Es posible que vengan sólo abogados a la asamblea.

c. \#Sólo los abogados son tales que es posible que vengan a la asamblea ${ }^{21}$.

${ }^{21}$ En casos como (25c), me veo en la necesidad de introducir un determinante en la paráfrasis buscada (los abogados). Esto es así porque no es posible dejar un plural escueto como sujeto preverbal del predicado de nivel individual que le sigue. Escojo el determinante definido porque, al tampoco tener alcance variable, considero que es el que interfiere menos en las condiciones de verdad de la paráfrasis buscada. 
(27) a. A la asamblea posiblemente vendrá un abogado.

$b$. Es posible que venga algún abogado a la asamblea.

c. Hay un abogado en particular que es posible que venga a la asamblea.

En suma, las frases nominales con puros tienen la propiedad de alcance inerte que tienen los plurales escuetos, tanto frente a la negación como frente a los cuantificadores y los operadores modales.

\section{Comportamiento anafórico}

Las frases nominales escuetas no pueden recibir una interpretación anafórica, y lo mismo sucede con las frases nominales introducidas por puros. En (28a) muestro un contexto en el que la frase indefinida muchas cartas introduce un referente plural que es retomado en (28b) por la frase definida las cartas. Esta conexión anafórica no se establece mediante una frase plural escueta, como la de (28c), ni tampoco por la frase nominal con puros en $(28 d)$, pues las cartas a las que se hace referencia en esas oraciones no son las mismas que las mencionadas en (28a):

(28) a. El fin de semana escribí muchas cartas.

b. Mañana voy a enviar las cartas.

c. Mañana voy a enviar cartas.

d. Mañana voy a enviar puras cartas.

Si bien las frases nominales indefinidas típicamente introducen referentes nuevos en el discurso ${ }^{22}$, las que tienen interpretación "fuerte" o partitiva, lo hacen relacionando los referentes nuevos con un conjunto mencionado previamente, o "anclado" en el universo discursivo ${ }^{23}$. Ese sería el caso si la oración (28a) tuviera una continuación como la de (29), en donde la frase nominal algunas cartas se puede interpretar como 'algunas de las cartas que escribí el fin de semana’:

${ }^{22}$ Irene Heim, The semantics of definite and indefinite noun phrases, Garland, New York-London, 1988.

${ }^{23}$ GARY Milsark, Existential sentences in English, tesis doctoral, Massachusetts Institute of Technology, Cambridge, MA, 1974; Kuaus von Heusinger, "Specificity and definiteness in sentence and discourse structure", JSem, 19 (2002), 245-274. 
(29) Mañana voy a enviar algunas cartas.

Lo que estos ejemplos muestran es que, una vez más, las frases nominales con puros se asemejan a los plurales escuetos en su incapacidad de recuperar un antecedente previamente establecido, y en esto contrastan tanto con las frases nominales definidas como con las frases indefinidas que pueden tener una interpretación "fuerte" o partitiva.

En los siguientes ejemplos mostraré que, además de no recuperar antecedentes, puros tampoco puede introducir referentes que se puedan retomar en una oración elíptica. Para ver esto, atendamos los ejemplos en (30). En las oraciones elípticas de estos ejemplos, el objeto del verbo se puede interpretar como correferente con el objeto de la oración coordinada que la precede. Es decir, tanto en (30a) como en (30b) se puede entender que las mantarrayas que vio Pablo fueron las mismas que vio Gustavo:

(30) a. Gustavo vio unas mantarrayas y Pablo también.

b. Gustavo vio mantarrayas y Pablo también.

En (31), esa interpretación se ve forzada por la presencia del clítico de objeto, cuyo antecedente anafórico se encuentra en el objeto de la primera oración coordinada:

(31) a. Gustavo vio unas mantarrayas y Pablo también las vio.

b. Gustavo vio mantarrayas y Pablo también las vio.

La accesibilidad de un antecedente para el clítico de objeto en (31a) está facilitada por la función típica de los determinantes indefinidos, que es la de introducir referentes nuevos que posteriormente se pueden recuperar con una expresión anafórica. En (31b), la primera oración coordinada no tiene como objeto una frase nominal indefinida, pero dado que el predicado es episódico, se asume que un cuantificador existencial "cierra" la proposición, y con ello se asume la existencia de alguna(s) mantarrayas que vio Gustavo y que serán las mismas que recupere el clítico de objeto en la oración siguiente. Otra posibilidad $^{24}$ es que el pronombre en $(31 b)$ exprese una anáfora a nivel de clase. En el siguiente ejemplo veremos que ninguna de estas

${ }^{24}$ Como señala un dictaminador anónimo. 
posibilidades anafóricas está disponible para las frases encabezadas por puros.

En (32), la frase puras mantarrayas no introduce un posible antecedente para el clítico anafórico de objeto, como sí lo hacen las frases indefinidas, ni tampoco legitima el cierre existencial automático que garantiza la accesibilidad de un antecedente, como hace el plural escueto. El resultado es que la oración con el clítico de objeto (32b) resulta agramatical, pues no tiene antecedente. La única lectura posible para $(32 a)$ es que la oración elíptica se interprete como correferente con todo el predicado de la oración que la precede, es decir, como 'Pablo también vio puras mantarrayas':

(32) a. Gustavo vio puras mantarrayas y Pablo también.

b. *Gustavo vio puras mantarrayas y Pablo también las vio.

Podemos concluir que las frases nominales con puros no son referenciales, pues ni recuperan ni necesariamente introducen referentes individuales que se puedan recuperar posteriormente. $\mathrm{Al}$ igual que las frases nominales escuetas, las frases nominales con puros tienen alcance inerte respecto de operadores como la negación, los cuantificadores y los modales. Las frases nominales introducidas por determinantes o cuantificadores indefinidos, en cambio, pueden tomar alcance variable respecto de este tipo de operadores.

Es pertinente considerar que algunos cuantificadores indefinidos pueden expresar matices que condicionen sus posibilidades de tomar alcance amplio (como algunos) o que les impida tomar alcance estrecho (como cierto), según se reporta en la bibliografía ${ }^{25}$. Por ello, sólo he mostrado contrastes entre las frases nominales con alcance inerte y las frases nominales indefinidas menos marcadas, como las introducidas por cardinales o el artículo indefinido.

Del comportamiento que he descrito se puede concluir una de las siguientes dos cosas: que puros no tiene una función de determinante/cuantificador, pues su presencia no tiene efecto sobre las propiedades de alcance inerte de un plural escueto, o bien

25 Sobre las restricciones de alcance de algunos, véase Luisa MarTí, "Restoring indefinites to normalcy", JSem, 24 (2007), 1-25. Sobre el alcance amplio de cierto, véase EGUREN y SÁNCHEZ, art. cit.; aunque un análisis divergente se encuentra en J. García FAJARdo, "El valor de cierto como determinante", Verba, 41 (2014), 1-16. 
que puros es un cuantificador indefinido, pero que está marcado para tener siempre alcance bajo. Esta última opción no parece desatinada, pues es un camino de gramaticalización bien conocido el que lleva a algunos adjetivos a convertirse en algún tipo de determinante ${ }^{26}$. En la sección siguiente, presentaré pruebas que sugieren que puros estaría en un estado avanzado de este proceso, pues tiene varias características de determinante que otros adjetivos de sentido cuantificativo no tienen.

\section{Puros Como Determinante: DisTribución SINTÁCtica}

En la sección previa mostré que las frases nominales con puros tienen las propiedades de alcance inerte y la falta de referencialidad que los asemejan a los plurales escuetos. Ahora mostraré que su distribución sintáctica, en cambio, es más parecida a la de las frases nominales cuantificadas. Consideraré, siguiendo a Gutiérrez Rodríguez y a Leonetti ${ }^{27}$, entre otros, que los cuantificadores adnominales son un tipo de determinantes ${ }^{28}$. Es claro que, por lo menos en español, varios de los que consideramos cuantificadores adnominales tienen su origen en adjetivos de sentido cuantificativo, como es el caso de varios y bastantes, que se pueden considerar cuantificadores determinantes en sentido pleno, y también el de numerosos, diversos y determinados, cuyo paso a una categoría funcional de determinante no se ha completado todavía ${ }^{29}$.

Varias pruebas que expondré a continuación sugieren que puros es un determinante del tipo de los cuantificadores, al menos si se consideran los dos siguientes aspectos de las frases nominales en las que aparece, que son características de las frases con determinante: 1) su función como sujetos preverbales y 2) su función como argumentos de predicados de nivel indivi-

26 M. LeOnetti, Los cuantificadores, p. 33.

27 Edita Gutiérrez Rodríguez, "Rasgos categoriales de los determinantes", en Actas del XXXVII Simposio Internacional de la Sociedad Española de Lingüistica (SEL)...; M. LeONETTI, Los cuantificadores.

28 Esta postura, aunque común, no está exenta de debate. Por ejemplo, GiUliana GiUsti considera que los cuantificadores son una clase sintáctica independiente de la de los determinantes ("The categorial status of determiners", en The new comparative syntax, ed. Liliane Haegeman, Cambridge University Press, Cambridge, 1997).

29 M. Leonetti, Los determinantes, Arco/Libros, Madrid, 1999, p. 22. 
dual. Comparten estas características las frases nominales con adjetivos prenominales como diversos y determinados, e incluso con calificativos antepuestos, por lo que no permiten reconocer si puros se ha recategorizado en un determinante. Por eso, en la segunda parte de esta sección mostraré que puros tiene más rasgos de determinante cuantificador que otros adjetivos, particularmente en la legitimación de categorías vacías como complemento nominal y la posibilidad de aparecer en estructuras partitivas.

Contrastes en la distribución de las frases nominales con puros y los plurales escuetos

Sujetos preverbales. Una característica fundamental de las frases determinantes en español es que pueden aparecer en posición de sujeto preverbal ${ }^{30}$. Si bien las frases nominales con puros en función de sujeto suelen estar pospuestas al verbo, es posible encontrarlas también antepuestas, como se muestra en (33a) y $(34 a)$. En los incisos (b) de número muestro que un sujeto sin determinante, en el mismo contexto, no puede ocupar la posición preverbal. Si el plural escueto tiene alguna prominencia prosódica, sí puede aparecer antes del verbo; por ejemplo, cuando introduce un tópico de contraste o un foco correctivo. Pero para comparar las frases nominales con puros con los plurales escuetos, es necesario no otorgar ninguna prominencia a la frase nominal en cuestión:

(33) a. Aseguró que con excepción de su protegido, el secretario general del partido..., "puros burócratas manejan al PRI” (CREA, 1996, Prensa, Proceso, 15/09/1996).

$b$. *Aseguró que burócratas manejan al PRI.

(34) a. Puras casitas pequeñas había antes (CECM, 1966, Anónimo, Cinta 2-I-B).

b. *Casitas pequeñas había antes.

${ }^{30}$ La posición menos marcada para los sujetos introducidos por puros, al menos a juzgar por la frecuencia de apariciones en CREA, es la postverbal. Sin embargo, las pruebas sintácticas que aquí se aplican no toman en cuenta la frecuencia con la que ocupan esa posición, sino simplemente si las oraciones son gramaticales o no lo son, según el juicio de un hablante nativo. 
Debemos considerar que, aunque los plurales escuetos no son aceptables como sujetos preverbales, la inaceptabilidad decrece considerablemente cuando se encuentran modificados por un adjetivo antepuesto, incluso si éste no es cuantificativo. Los siguientes ejemplos serían, pues, aceptables -aunque quizás sólo en un registro formal:

(35) Hábiles burócratas manejan el partido.

(36) Miserables casitas pueblan el paisaje.

En suma, puros legitima sujetos escuetos en posición preverbal, lo que se ha considerado por excelencia el papel de los determinantes ${ }^{31}$. Antes de saltar a la afirmación de que, por esta sola prueba, puros es un determinante, se debe recordar que los adjetivos calificativos antepuestos, como en (35) y (36), también legitiman una frase nominal escueta en esa posición, sin que ello nos lleve a decir que adjetivos como miserable o hábil sean determinantes. Por lo pronto, teniendo en cuenta estos datos, presentaré más contextos en los que las frases nominales con puros tienen un comportamiento similar al de las frases con determinante, y posteriormente sopesaremos las consecuencias de asignarle esta categoría sintáctica.

Argumentos de predicados de nivel individual. Los plurales escuetos no pueden ser argumentos de predicados de nivel individual. Si el sustantivo está modificado por un adjetivo, se aminora la inaceptabilidad y desaparece del todo si el argumento está introducido por un adjetivo cuantificativo o si es una frase nominal con puros:

(37) Contexto: Estamos buscando un regalo de cumpleaños para nuestra amiga Martha, que es bióloga.

a. *A Martha le gustan peces.

b. A Martha le gustan diversos peces /??extraños peces.

c. A Martha le gustan puros peces.

31 E. Gutiérrez Rodríguez, art. cit.; Ignacio Bosque, "Por qué determinados sustantivos no son sustantivos determinados", en El sustantivo sin determinación. La ausencia de determinación en la lengua española, ed. cit., pp. 13-119; véase también M. LeOnetTi, Los determinantes. 
(38) a. *Martha odia (a) diseñadores italianos.

b. Martha odia (a) diferentes diseñadores italianos/?famosos diseñadores italianos.

c. Martha odia a puros diseñadores italianos.

Los contrastes mostrados en (37) y (38), entre plurales escuetos en $(37 a)$ y $(38 a)$ y frases nominales con puros en $(37 c)$ y (38c), muestran que puros legitima una frase nominal como argumento de predicados individuales. Sin embargo, la aceptabilidad marginal de las frases con adjetivos prenominales en $(37 b)$ y $(38 b)$ nos hace pensar un poco antes de concluir que, por este motivo, puros sea un determinante.

En suma, hemos visto que las frases nominales con puros pueden ocupar posiciones sintácticas que están vedadas a los plurales escuetos: sujetos preverbales y argumentos de predicados de nivel individual. Se puede argumentar que las frases nominales escuetas con adjetivos antepuestos, incluso si no son cuantificativos (grandes empresarios, futuros periodistas) tienen una distribución parecida y que, por lo tanto, las pruebas que he expuesto aquí no son suficientes para caracterizar a puros como un determinante. En las siguientes secciones, describiré algunos contrastes entre las frases nominales con puros y las frases con adjetivos antepuestos. Me concentraré especialmente en adjetivos cuantificativos, para señalar que puros, en comparación con otros de estos adjetivos, tiene algunas de las propiedades sintácticas características de un determinante cuantificador.

Contrastes entre las frases nominales con puros y las frases nominales escuetas con adjetivos antepuestos

Otra característica de los determinantes, según Gutiérrez Rodríguez $^{32}$, es la de legitimar categorías vacías como complemento. En (39), se muestra que un cuantificador como tres o todos puede legitimar la categoría vacía representada como $e$. Los adjetivos prenominales como diversos y determinados, a pesar de que pueden introducir frases nominales como sujetos preverbales, no legitiman categorías vacías, según se muestra en (40). Según esta prueba, puros no sería un determinante, como se ve por la agramaticalidad de (41):

32 E. Gutiérrez Rodríguez, art. cit., p. 299. 
(39) En el bazar había pantalones de pana. Compré tres/todos e.

(40) En el bazar había pantalones de pana. *Compré diversos/ numerosos e.

(41) En el bazar había pantalones de pana. *Compré puros e.

Sin embargo, si a la categoría vacía le sigue un modificador, puros parece comportarse según el patrón de los determinantes y no el de los adjetivos:

(42) a. En la feria había pantalones de pana. Compré puros e morados.

b. En la feria había pantalones de pana. *Compré diversos/ numerosos e morados.

(43) $a$. Bueno, sigo con el especial de canciones... Estoy poniendo puras e que me gustan (Google, 02-10-14).

b. *Estoy poniendo diversas/numerosas e que me gustan ${ }^{33}$.

Otro argumento a favor de que puros es un determinante cuantificador es que puede tener un uso partitivo ${ }^{34}$. En esto,

33 Un dictaminador anónimo considera que (43b) es gramatical, por lo que sugiere que se someta este tipo de construcciones al juicio de otros hablantes. En una encuesta informal, pregunté a hablantes de español de México si consideraban aceptable o inaceptable las siguientes tres opciones (el contexto es el de un disc jockey que está poniendo boleros en un programa especial): 1) Estoy poniendo puros que me gustan; 2) Estoy poniendo numerosos que me gustan y 3) Estoy poniendo diversos que me gustan. De las 32 personas que juzgaron las tres oraciones, 2 juzgaron 1) inaceptable contra 30 que la consideraron aceptable; respecto a 2), 28 consideraron que era inaceptable, mientras que 4 la consideraron marginalmente aceptable. Respecto a 2), 26 consideraron que era inaceptable, mientras que 6 juzgaron que era medianamente aceptable. El contraste más claro fue que ninguno de los 32 consideraron ni 2) ni 3) completamente aceptables.

34 Se puede encontrar a diversos y a numerosos en estructuras partitivas: Las compañias telefónicas ofrecen diversos de esos servicios; Los Rotarios se sumarán a diversos de esos proyectos; Numerosos de esos reclusorios se encuentran supersaturados de inquilinos. Al parecer, éste es un aspecto en el que estos adjetivos cuantificativos tienen comportamiento de cuantificadores, mientras que otros adjetivos, que no tienen origen cuantificativo, no aceptan terminaciones partitivas: *Ofrecen ciertos de esos servicios (LEONETTI, Los cuantificadores, p. 46). Lo interesante es que puros, sin ser originalmente un adjetivo cuantificativo, sí puede entrar en estructuras partitivas, como un cuantificador. 
puros se asemeja a otros adjetivos claramente cuantificacionales, como bastantes y varios:

(44) Veía el televisor y le decía a mi mamá que quería ser un jugador de raza negra. Había puros de esos en la NBA y Michael Jordan me impresionó (Google, 02-10-14).

(45) Y puros de esos atropellos se registraron en este festejo ya ni tan familiar (Google, 02-10-14).

Resumo lo expuesto en esta sección: las frases nominales con puros contrastan con las frases nominales escuetas sin modificación en que pueden aparecer como sujetos preverbales y pueden ser argumentos de predicados de nivel individual. Otros adjetivos cuantificativos, como numerosos y diversos (e incluso algunos calificativos prenominales), también cumplen esos criterios. Sin embargo, de acuerdo con otras pruebas, puros se comporta de manera más parecida a los adjetivos cuantificacionales como varios y bastantes, cuya recategorización como determinantes se considera completa $^{35}:$ 1) puros legitima categorías vacías siempre y cuando vayan seguidas de un modificador adjetival o cláusula relativa (en contraste con numerosos o diversos), 2) puros puede entrar en construcciones partitivas, como un auténtico cuantificador (por oposición a otros determinantes de origen adjetival, como cierto).

Según la distribución sintáctica hasta aquí presentada, cabe concluir, por lo menos provisionalmente, que puros es un adjetivo que se ha recategorizado como un determinante cuantificador. Este tipo de recategorizaciones es bastante común, sobre todo cuando el adjetivo tiene sentido cuantificativo, pero se llega a dar también con adjetivos calificativos que en posición prenominal funcionan como determinantes (aunque no necesariamente como cuantificadores). En la siguiente sección, describiré la estructura de los cuantificadores adnominales y el problema que enfrentamos al adscribir a puros dentro de esta categoría. 
Desde el punto de vista de su estructura lógica, un cuantificador es una expresión que relaciona dos conjuntos. En la oración Algunos gatos muerden, el determinante existencial algunos se usa para afirmar que la intersección del conjunto de los gatos y el conjunto de los individuos que muerden no está vacía. Al primero de los conjuntos (en nuestro ejemplo, el conjunto de los gatos) se le conoce como restrictor y al segundo (el de los individuos que muerden) como alcance nuclear. En una oración como Todos los gatos muerden, el cuantificador universal expresa que el conjunto de los gatos (el restrictor) es un subconjunto del de los individuos que muerden.

Una característica de los cuantificadores nominales es que su restrictor siempre corresponde a la denotación de la frase nominal con la que se combina. A esta correspondencia entre el complemento sintáctico del determinante y el restrictor semántico del cuantificador que denota se le conoce como conservatividad ${ }^{36}$.

Si puros es un determinante cuantificador, las condiciones de verdad de una oración como (46a) podrían formalizarse como en (46b), que a su vez se parafrasea de manera informal en $(46 c)$ :

(46) a. Hay puros gatos en el asilo.

b. $\forall \mathrm{x}$ (está-en-el-asilo $(\mathrm{x}) \rightarrow$ gato $(\mathrm{x}))$.

c. Todos los individuos ${ }^{37}$ que hay en el asilo son gatos.

La fórmula en $(46 b)$ y su parafraseo en $(46 c)$ capturan las condiciones de verdad de $(46 a)$ de manera satisfactoria. La expresión puros correspondería, pues, a un cuantificador universal y la oración entera es una afirmación acerca de la totalidad de entidades que hay en el asilo, de las que se predica que son gatos. El lector habrá notado, sin embargo, que en (46b), la

${ }^{36}$ E. Keenan y J. Stavi, art. cit., aunque primero fue propuesta por JoHn BAR wise y Robin CoOper bajo el término live-on property, en "Generalized quantifiers and natural language”, LiP, 4 (1981), 159-219.

37 Estoy dando por hecho que toda cuantificación en uso viene con una restricción contextual. Por lo tanto, $(46 a)$ y (46b) son verdaderas incluso si en el asilo, además de gatos, hay ventanas, muebles, personas, etc., es decir, un escenario donde no estrictamente todos los individuos son gatos. Esto es posible porque se asume un dominio relevante, por ejemplo, el de los animales. Así, el enunciado En el asilo hay puros gatos o Todo lo que hay en el asilo son gatos, implícitamente quiere decir Todos los animales que hay en el asilo son gatos. 
restricción del cuantificador universal -el conjunto dentro del cual la variable x encuentra su valor- es el conjunto de individuos que están en el asilo y no el conjunto de los gatos. Esto es problemático, porque el complemento sintáctico de puros es el sustantivo gatos, y el conjunto que este sustantivo denota, precisamente, no está actuando como restrictor del cuantificador universal correspondiente a puros. En conclusión: si analizamos puros como un cuantificador, inevitablemente enfrentamos el problema de su no-conservatividad.

Hay buenas razones para pensar que todos los cuantificadores nominales de las lenguas naturales son conservativos. Es plausible pensar que los determinantes de las lenguas se restringen a los que expresan funciones conservativas, al menos por dos razones: una de índole semántica composicional y otra de carácter pragmático-discursivo. Del lado de la semántica composicional, la conservatividad garantiza que la interpretación de las expresiones complejas proceda a la par de su estructura sintáctica. Si un cuantificador nominal se combina primero que nada con un sustantivo, ese sustantivo también es su argumento lógico, es decir, su restricción, de modo que el cuantificador no busca su restricción aleatoriamente en otros constituyentes de la oración. Desde el punto de vista discursivo, la conservatividad garantiza que el sustantivo que acompaña al cuantificador nos remita consistentemente a "aquello de lo que estamos hablando", por decirlo de alguna manera. En palabras de Westerståhl ${ }^{38}$, la conservatividad asegura que el universo se restrinja a la denotación del sustantivo, mientras que la frase verbal proporcione lo que se predica acerca de aquello de lo que hablamos. Así, sabemos que la oración algunos gatos muerden afirma algo sobre los gatos, no sobre los individuos que muerden, ni sobre todos los individuos del universo. Sin el principio de conservatividad de los determinantes, esta correspondencia no sería consistente y la interpretación, por consiguiente, sería más costosa.

En suma, la propiedad de conservatividad es un universal que vale la pena considerar en los cuantificadores y, probablemente, la faceta más importante de puros como cuantificador es que desafía este universal de manera patente.

Propondré, por lo tanto, un análisis que no implique abandonar el principio de conservatividad en favor de considerar puros un determinante. Después de todo, a pesar de su distri-

38 Art. cit. 
bución sintáctica, en la segunda sección expuse la evidencia de que puros tiene alcance inerte ante otros operadores, lo que ya pone en duda su estatus como operador.

\section{Puros es un modificador nominal}

Si queremos evitar considerar puros como un determinante, la única alternativa que nos queda es considerarlo un adjetivo -pues ya mostré que su concordancia de número y género, además de su posible flexión de diminutivo, evidencian que no se trata de un adverbio. Como adjetivo, puros introduce una propiedad: la denotada por el sustantivo que lo acompaña. Pero en lugar de que esa propiedad exprese el rango de una variable (es decir, la restricción de un cuantificador), simplemente constituye una propiedad que se predica acerca de un individuo plural. El individuo plural es el conformado por la suma total de los individuos que satisfacen el predicado provisto por el resto de la oración. De este modo, la oración (46a), repetida como $(47 a)$, se puede parafrasear como en $(47 b)$ y queda capturada formalmente en $(47 c)$ :

(47) a. Hay puros gatos en el asilo.

b. La propiedad 'ser gato' es verdadera de la suma total de individuos que hay en el asilo.

c. $\lambda \mathrm{P} \imath \mathrm{Q} . \forall \mathrm{x}(\mathrm{x} \leq(\mathrm{1Q}) \rightarrow \mathrm{P}(\mathrm{x}))($ gatos $)$ (hay-en-el-asilo).

d. $[[$ puros $]]=\lambda \mathrm{P} 1 \mathrm{Q} \cdot \forall \mathrm{x}(\mathrm{x} \leq(1 \mathrm{Q}) \rightarrow \mathrm{P}(\mathrm{x}))$.

El contenido semántico de puros equivale a una función compleja, representada en $(47 d)$ y que se puede explicar como sigue: es una función que toma como argumento una propiedad $(\mathrm{P})$ y da como valor un individuo $(\mathrm{lQ})$, tal que todas sus partes $(\mathrm{x})$ tienen la propiedad $(\mathrm{P})$. La propiedad $(\mathrm{P})$ es el primer argumento semántico de puros y corresponde a su primer argumento sintáctico (el sustantivo gato, en $50 a$ ), por lo que su computación semántica procede a la par de su derivación sintáctica, es decir, de manera composicional.

Respecto al individuo plural sobre cuyas partes se predica la propiedad de ser gato, éste se obtiene al aplicar el operador 1 al conjunto proporcionado por el resto de la oración. El operador 1 tiene el efecto de convertir un conjunto en un individuo, 
al seleccionar su suma máxima, que se entiende como un individuo plural (o singular, si el conjunto es unitario).

De este modo, puros no tiene propiamente la semántica de un cuantificador, porque no relaciona dos conjuntos, sino que relaciona un conjunto con un individuo. De esa manera, tendría el tipo semántico de los modificadores y la categoría sintáctica de los adjetivos, que son los elementos que típicamente cumplen la función de modificación.

\section{Expresiones similares en otras lenguas}

El alemán "lauter". El análisis que propongo en $(47 d)$ está basado en el propuesto por Eckardt ${ }^{39}$ para la expresión alemana lauter, que tiene un origen etimológico similar al de puro(s), y un uso contemporáneo también parecido ${ }^{40}$. Eckardt reconoce el problema de la no-conservatividad de lauter, si se le considera un determinante, y propone un análisis como modificador, que reproduzco en (49):

(48) Susi aß lauter Birkenpilze.

Susi comió sólo especie-de-hongo.

'Susi comió puros champiñones' 41 .

(49) a. $[[$ lauter $]]=\lambda \mathrm{P} \lambda \mathrm{X} . \forall \mathrm{x}(\mathrm{x} \leq \mathrm{X} \rightarrow \mathrm{P}(\mathrm{x}))$.

b. $\lambda \mathrm{P} \lambda \mathrm{X} . \forall \mathrm{x}(\mathrm{x} \leq \mathrm{X} \rightarrow \mathrm{P}(\mathrm{x}))$ (champiñones) (comió-Susi).

Lo que refleja la fórmula en $(49 a)$ es que lauter se combina con una propiedad -la denotada por el sustantivo con el que

39 En op. cit.

40 Con la excepción de que lauter, según refiere la autora, implica que las entidades involucradas son muchas. Hay un uso parecido de puros en español mexicano, pero los casos que aquí analizo no llevan necesariamente esa implicación de alta cardinalidad. Por ejemplo, para R. ECKARDT (p. 206), la oración 1) es inadecuada si los Maier tienen sólo dos hijas, mientras que la traducción con puros que ofrezco es perfectamente adecuada en ese escenario y la oración es verdadera siempre y cuando los Maier no tengan hijos varones:

1) Die Maiers haben lauter Töchte.

los familia-Maier tienen sólo hijas.

'Los Maier tienen puras hijas'.

${ }^{41}$ Me estoy tomando la libertad de traducir Birkenpilze por 'champiñones', aunque en realidad se trata de un hongo del género boletus, cuyo nombre en español mexicano desconozco. 
lauter forma una frase nominal (Birkenpilze) - y predica que esa propiedad es verdadera de todas las partes atómicas que conforman a un cierto individuo plural (X), que a su vez, es algo que comió Susi.

Sin embargo, hay un aspecto crucial sin el cual la fórmula en (49a) no puede representar las condiciones de verdad de una oración como (48), a saber: para que (48a) sea verdadera no es suficiente que haya una pluralidad de objetos que comió Susi y cuyos miembros sean todos champiñones. Debe ser el caso que la totalidad de las cosas que comió Susi tengan la propiedad de ser champiñones. Voy a llamar a esa implicación condición de maximalidad.

Eckardt deja fuera de la representación veritativa de lauter la referencia a esta totalidad, y la incluye dentro de la representación semántica por medio de una restricción contextual. Para Eckardt, la condición de maximalidad no es parte del contenido veritativo de lauter, sino efecto de una restricción contextual que requiere que la totalidad de individuos relevantes sea considerada como partes de la pluralidad en cuestión. Cada contexto encontrará una instanciación distinta de lo que se considera "relevante" 42 .

Creo que extender este análisis a puros no captaría el hecho crucial de que la condición de maximalidad que introduce puros sí es parte de su contenido veritativo. Para que la oración española Susi comió puros champiñones sea verdadera se requiere no sólo que Susi haya comido una pluralidad de objetos cuyos miembros son todos champiñones, sino, además, que esa pluralidad sea todo lo que comió, que no haya en el conjunto de cosas que comió nada fuera de esa pluralidad. Este entrañamiento es fuerte y necesita reflejarse en las condiciones de verdad, pues si no se satisface, la oración es abiertamente falsa y no sólo "inadecuada" o "infeliz" por infringir restricciones contextuales.

42 La fórmula de ECKARDT, con la restricción contextual, quedaría como sigue:

1) $[[$ lauter $]]=\lambda \mathrm{P} \lambda \mathrm{X} \cdot \forall \mathrm{x}(\mathrm{x} \leq \mathrm{X} \rightarrow \mathrm{P}(\mathrm{x}))$

b. $\lambda \mathrm{X}$. $[\forall \mathrm{x}($ Relevante $(\mathrm{x}) \rightarrow \mathrm{x} \leq \mathrm{X}) \wedge \forall \mathrm{x}(\mathrm{x} \leq \mathrm{X} \rightarrow \mathrm{P}(\mathrm{x}))]$

No incluyo otra restricción contextual añadida por R. ECKARDT (op. cit.) al significado de lauter, a saber 'Grande(X)', que requiere que el tamaño de la pluralidad sea considerable. Este significado no es parte de las implicaciones necesarias de puros, y no es relevante en la comparación de la expresión alemana con la española. 
Por esa razón he decidido adaptar el análisis de Eckardt introduciendo el operador 1 , que directamente extrae una pluralidad a partir del predicado provisto por el resto de la oración, sin necesidad de postular restricciones contextuales externas a las condiciones de verdad.

Inglés: "few" y "many". Compárense los siguientes ejemplos, propuestos por Westerståhl ${ }^{43}$ :

(50) Many winners of the Nobel Prize in literature are Scandinavians.

'Muchos ganadores del Premio Nobel de literatura son escandinavos'.

(51) Many Scandinavians have won the Nobel Prize in literature. 'Muchos escandinavos han ganado el Premio Nobel de literatura'.

(52) Many Scandinavians are Nobel Prize winners in literature. 'Muchos escandinavos son ganadores del Premio Nobel de literatura'.

Claramente, las condiciones de verdad de (50) y de (52) no son las mismas. Mientras que en el mundo actual (50) es verdadero, (52) no lo es. En cambio, (51) es ambigua: en una de sus interpretaciones es equivalente a (50) y en la otra, a (52). Es especialmente interesante la interpretación de (51), en la que es equivalente a (50), pues en ese caso, aunque Scandinavians sea la frase nominal que contribuye el primer argumento sintáctico de many, su restrictor realmente parece aportarlo la frase verbal, have won the Nobel Prize in literature. En otras palabras, many en esa interpretación cuantifica sobre los ganadores del premio Nobel y no sobre los escandinavos. Puesto que el restrictor del cuantificador no es su primer argumento sintáctico, se puede considerar que many, por lo menos en esa lectura, es un cuantificador no-conservativo.

Otro caso de determinantes aparentemente no conservativos en inglés es el estudiado por H. Herburger ${ }^{44}$ :

(53) Few incompetent cooks applied.

'Pocos cocineros incompetentes concursaron'.

43 Art. cit., p. 403.

${ }^{44}$ H. Herburger, op. cit., pp. 123-139. 
(54) Few INCOMPETENT cooks applied.

'Concursaron pocos cocineros INCOMPETENTES'.

La oración (53) en inglés es ambigua: en una de sus interpretaciones (la lectura proporcional, o "fuerte", también llamada partitiva), describe una situación en la que, de los cocineros incompetentes, concursaron pocos. En la otra interpretación (la "débil" o simétrica), sólo se afirma que el conjunto de individuos que son cocineros incompetentes y que concursaron tiene una cardinalidad baja.

En contraste, la oración (54) y su traducción al español no son ambiguas, sino que describen una situación en la que, del universo de cocineros concursantes, un número muy pequeño era de cocineros incompetentes. Como se ve en esa posible paráfrasis, la restricción del cuantificador few no es el conjunto de los cocineros incompetentes (como en 53), sino el de los cocineros que concursaron. El alcance nuclear del cuantificador está conformado por el conjunto denotado por el adjetivo en foco.

Herburger (id.) nota que otros cuantificadores cardinales (incluido el many de Westerståhl) tienen esta facultad de interpretarse de acuerdo con la estructura focal de la oración, dando lugar a lo que llama lecturas afectadas por foco. Estas lecturas se deben a una regla, llamada proyección de foco (focus-mapping), que asocia el alcance nuclear de un cuantificador con el constituyente oracional que está en foco, aunque éste no corresponda al primer argumento sintáctico del determinante. El resto de la oración, la información no focal, constituye la restricción. Se trata, pues, de determinantes conservativos, en última instancia, sólo que para "salvar" la conservatividad hay que recurrir a la regla de proyección de foco y no directamente a la estructura sintáctica de superficie.

Con la regla de proyección de foco, la estructura cuantificacional no depende de la estructura sintáctica superficial, sino de un acomodo que tiene lugar en una estructura sintáctica alternativa. Una de las razones por las cuales consideramos que el principio de conservatividad es valioso como universal es porque nos permite relacionar directamente la estructura de la frase determinante y la interpretación del cuantificador. Relegar esta relación a una forma sintáctica distinta de la superficial no rescata la conservatividad del cuantificador, pues, de ese modo, 
cualquier cuantificador termina siendo conservativo, trivializando así la noción de conservatividad.

Además, una parte crucial del análisis de Herburger es que la regla de proyección focal sólo se pueda ejecutar con los determinantes "débiles", como few y many. Esto acarrea un problema empírico, pues en inglés y en italiano se pueden obtener lecturas aparentemente no conservativas con los determinantes "fuertes" ally tutti, respectivamente, cuando aparecen en contextos episódicos ${ }^{45}$, como los ejemplificados en (55) y (56):

(55) a. I have all boys, so I cannot compare how easy they are to girls $^{46}$.

'Tengo puros niños, por eso no puedo saber qué tan fáciles son en comparación con las niñas'.

$b$. There were all freaks in that café.

'Había puros raritos en ese café'.

(56) Io ho tutti bei ricordi, persino degli anni di Guerra.

'Tengo puros buenos recuerdos, incluso de los años de guerra'.

Las oraciones en $(55 a)$ y $(55 b)$ no predican algo acerca de todos los niños o de todos los "raros". Más bien, el nominal boys en ( $55 a$ ) se predica de todos los hijos que tiene el hablante, y el sustantivo freaks se predica de todos los individuos que estaban en el café. Lo mismo sucede con la oración (56): el hablante no afirma tener todos los recuerdos buenos, sino que dice, respecto de todos los recuerdos que tiene, que son buenos. Para obtener la interpretación que se refleja en las traducciones no es necesario que el sustantivo que sigue al cuantificador tenga un acento prominente, lo que sugiere que no necesariamente está en foco.

Si recapitulamos, además de lauter, podemos identificar los siguientes ejemplos de determinantes no conservativos:

(57) Few COOKS applied.

'Pocos COCINEROS concursaron'.

45 Agradezco esta observación a Anna Szabolcsi (c.p. 2009).

${ }^{46}$ Los ejemplos en (55) y (56) fueron tomados de Google y verificados, tanto para gramaticalidad como para interpretación, con hablantes nativos de inglés e italiano, respectivamente. 
(58) Many Scandinavians have won the Nobel Prize.

'Muchos escandinavos han ganado el premio Nobel'.

(59) I have all boys.

'Tengo puros niños'.

Todas estas oraciones son susceptibles del mismo análisis que proporcioné para las oraciones con puros. En cada caso, el "determinante" (few, many, all) cuantifica sobre partes de un individuo plural, que en todos ellos corresponde a la suma total de individuos que pertenecen al conjunto denotado por la oración menos la frase cuantificacional. Por ejemplo, en (57) el individuo plural es la suma máxima de individuos que concursaron; y de ellos la frase nominal predica que pocos eran cocineros. En (58), el individuo plural en cuestión es la suma máxima de individuos que han ganado el Premio Nobel y lo que la frase many Scandinavians predica es que muchas de sus partes (atómicas) son escandinavos. Por último, en (59) el individuo plural es la suma máxima de (hijos) que tengo y la frase all boys predica de todos ellos que son niños.

Todas estas expresiones (few, many, all, tutti, lauter) introducen una propiedad que se predica, ya sea de todas las partes de un individuo plural (en el caso de all, tutti, lauter, csupa), ya sea de pocas o muchas de ellas (el caso de few y many, respectivamente). Varían, pues, en si el cuantificador sobre partes es universal o un cardinal impreciso. El análisis propuesto salva el problema de la no-conservatividad al no considerarlos cuantificadores.

Las expresiones que, en distintas lenguas, tienen el significado actual de puros (ej. el alemán lauter, el húngaro csupa) ${ }^{47}$, tienen su origen en adjetivos con etimología similar, y no necesariamente en cuantificadores, por lo que forzar un análisis de estas expresiones en términos de cuantificadores no parece deseable. En cambio, considerar cuantificadores como few, many y all como modificadores goza de una tradición más aceptable. En suma, creo que lo que permite la interpretación aparentemente no conservativa de un cuantificador no es que éste sea "débil", sino, en última instancia, que pueda cumplir las funcio-

47 Agradezco a Anna Szabolcsi (c.p.) este dato del húngaro:

1) Csupa jó emlékem van.

‘desnudo' bueno recuerdo.poss.1sg está.

'Tengo puros buenos recuerdos'. 
nes de un modificador. Por esta razón, los cuantificadores most o every no pueden tener las lecturas aparentemente no conservativas que tienen many, few y all.

\section{Conclusiones}

Se ha mostrado que la expresión puros del español tiene el comportamiento de un cuantificador y a la vez de un modificador adjetival. Con base en un análisis similar propuesto por Eckardt ${ }^{48}$ para el alemán, propuse que puros es un modificador, uno de cuyos argumentos es un individuo plural que corresponde a la suma máxima de individuos denotada por el predicado. La expresión tiene un contenido cuantificacional, porque contiene un operador que cuantifica sobre las partes de ese individuo para predicar de ellas la propiedad denotada por el sustantivo, que es su primer argumento. Una diferencia de mi análisis con el de Eckardt es que, para mí, la condición de maximalidad es parte de la semántica veritativa de puros y no de una restricción contextual.

Además, comparé las condiciones de verdad de las oraciones con puros con otras expresiones similares en otras lenguas, particularmente con los llamados "determinantes no conservativos" del inglés: las lecturas afectadas por foco de Herburger ${ }^{49}$, el many no conservativo de Westerståhl ${ }^{50}$ y algunos usos de all en contextos existenciales. Mostré que tratar estas expresiones como modificadores en lugar de cuantificadores, de manera similar a como hicimos con puros, evita el problema de tener determinantes no conservativos. Coincidentemente, todas las expresiones que pueden tener lecturas que equivaldrían a la de un cuantificador no conservativo tienen una naturaleza adjetival, como es el caso de few y many que, a pesar de actuar como determinantes en inglés, también tienen usos como modificadores. Lo mismo sucede con all, cuantificador fuerte del que se ha argumentado que es más bien un modificador, pues precede a frases con determinante (all the boys) ${ }^{51}$. Parece, pues, que para completar su conversión de modificador a determinantes, un

$48 \mathrm{Op}$. cit.

${ }^{49} \mathrm{O} p$. cit.

${ }^{50}$ Art. cit.

51 Christine M. Brisson, "Plurals, all, and the non-uniformity of collective predication”, LiP, 26 (2003), 129-184. 
adjetivo primero debe garantizar que el determinante en cuestión sea conservativo. De otro modo, es susceptible de analizarse como modificador bajo las líneas que propuse en este trabajo.

Por último, un fenómeno más general que llama la atención es que los adjetivos prenominales en español se reinterpretan consistentemente como expresiones más funcionales que léxi$\operatorname{cas}^{52}$. Determinar exactamente qué tipos funcionales están disponibles en esa posición -y no solamente adscribirlos a una categoría amplia de "operadores"-, puede revelar mucho sobre la configuración sintáctica y semántica de la frase nominal en español. Vale la pena reconsiderar si la legitimación de frases nominales en posición de sujeto preverbal está realmente restringida a la presencia de un determinante o si hay expresiones de otros tipos funcionales, de los disponibles en la posición prenominal, que puedan tener este efecto.

Violeta Vázquez Rojas Maldonado
El Colegio de México

${ }^{52}$ Varias expresiones de este tipo se han estudiado en una serie amplísima de trabajos, entre los que remito al lector a los previamente mencionados: J. García Fajardo, "El valor de cierto como determinante"; J. Pozas Loyo, "Diacronía de cierto"; C. Fuentes Rodríguez "Los operadores argumentativos puro, mero y simple"; L. Eguren y A. FÁbregas, "Cuestiones de orden: semántica y sintaxis de los adjetivos ordinales", EstL,19 (2005), 85-105; B. CAmus Bergareche, "De adjetivos a cuantificadores. Un fragmento de la historia de los indefinidos románicos", entre otros. 